\title{
Ethnomedicobotany of Rajbanshi community in the Duars region of Jalpaiguri District, West Bengal, India
}

\author{
Dipanwita Debnath ${ }^{1}$, Baby Roy Barman ${ }^{2}$, Dibakar Choudhury ${ }^{3}$ \\ and A. P. Das \\ ${ }^{1}$ West Congress Para, Jalpaiguri - 735101, West Bengal, India \\ ${ }^{2}$ Vill.: Chaitu Para, P.O.: Talma (Jahuri) - 735121, Jalpaiguri, West Bengal, India \\ ${ }^{3}$ Vill. \& PO: Shilbarihat, Alipurduar - 736204, West Bengal, India \\ ${ }^{4}$ Department of Botany, Rajiv Gandhi University, Rono Hills, Doimukh-791112, Arunachal Pradesh, India \\ 'Communicating author, e-mail: dipannitadebnath1@gmail.com
}

[Received 09.05.2019; Revised 21.06.2019; Accepted 23.06.2019; Published 30.06.2019]

\begin{abstract}
The Rajbanshi community in Duars of Jalpaiguri district of West Bengal is traditionally using different kinds of plant parts as medicine. Present article discussed the medicinal plants used by them including different formulations. Leaves are the most used plant parts. However, their tradional knowledge is in danger as the facilities of modern civilization is spreading fast in the area.
\end{abstract}

Key word: Ethnobotany, Medicinal Plants, Rajbanshi Community, Duars, Jalpaiguri District

\section{INTRODUCTION}

Northern part of the state of West Bengal, India is covered with rich vegetation, mainly forested and quite a good number of tribal communities are living there since an unknown period of time. In Terai-Duars region some communities like Mech, Rabha, Toto, Santal, Oraon, Lepcha,Sherpa, Dukpa and Rajbanshi (Mukherjee et al. 2007; Sarkar 2011; Dennath et al. 2017) are living with quiet harmony in an forested environment. In West Bengal, Rajbanshi community has been placed in the list of scheduled caste (SC) category(www.rajbanshipride.blogspot.in/2016/02/social-issiuesscst-status-of-rajbanshi).

The traditional botanical knowledge of Rajbanshi community is very rich (Roy \& Das 2015; Debnath et al. 2017). They use plants in almost all aspects of their life. While Roy \& Das (2015) discussed the preparation of some of their special type of food-items, Debnath et al. (2017) discussed different aspects of their traditional foods.

Like the people of most of other communities, people of Rajbangshi community also uses many plant based traditional medicines to treat/cure their different diseases. However, Sanyal (1965) emphasised that ancient Rajbanshi people did not have knowledge about the medicinal uses of plant. So, when they were suffering from different types of physical discomforts of ailments like fever, cold cough, Andha sula (Night Blindness) etc, then at first they applied some "Mantra" expecting that the God will cure them through their enforced Mantras. Later on, gradually, they started applying some plant parts (leaf, stem, bark, root etc) through trial and error method. Sometimes they were successful and gradually they developed their own mainly plant-based medical system. After this success, these techniques are now being used traditionally for generations to cure these diseases. As for example, during fever Rajbangshi people used some kind of "Mantra" with or without any plant-based medicine: 


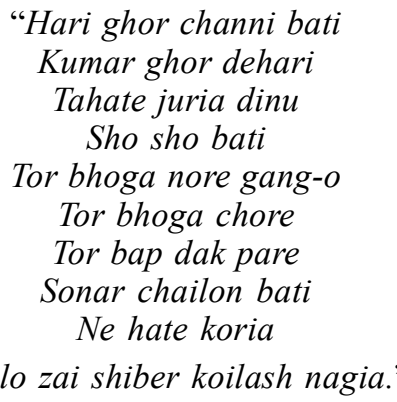

Till date the ethnomedicobotany of Rajbanshi people was not studied by any worker. As they are mainly concentrated in this Terai-Duars part of the country but are distributed in Indian states like assam, Arunachal Pradesh, Bihar and other countries like Bangladesh and Nepal (Sanyal 1965; Debnath et al. 2017).

\section{STUDY AREA}

All data are collected from the Rajbanshi dominating forest-villages in the Jalpaiguri district of West Bengal (India). The district is lying between $26^{\circ} 16^{\prime} \mathrm{N}$ to $27^{\circ} 0^{\prime} \mathrm{N}$ Latitudes and $88^{\circ}$ $4^{\prime} \mathrm{E}$ to $89^{\circ} 53^{\prime} \mathrm{E}$ longitudes with an average elevation of $75 \mathrm{~m}$ AMSL. It is surrounded by Bhutan in North, Bangladesh in South, Darjeeling Hills in the west and Alipurduar and Coochbihar districts on the east.

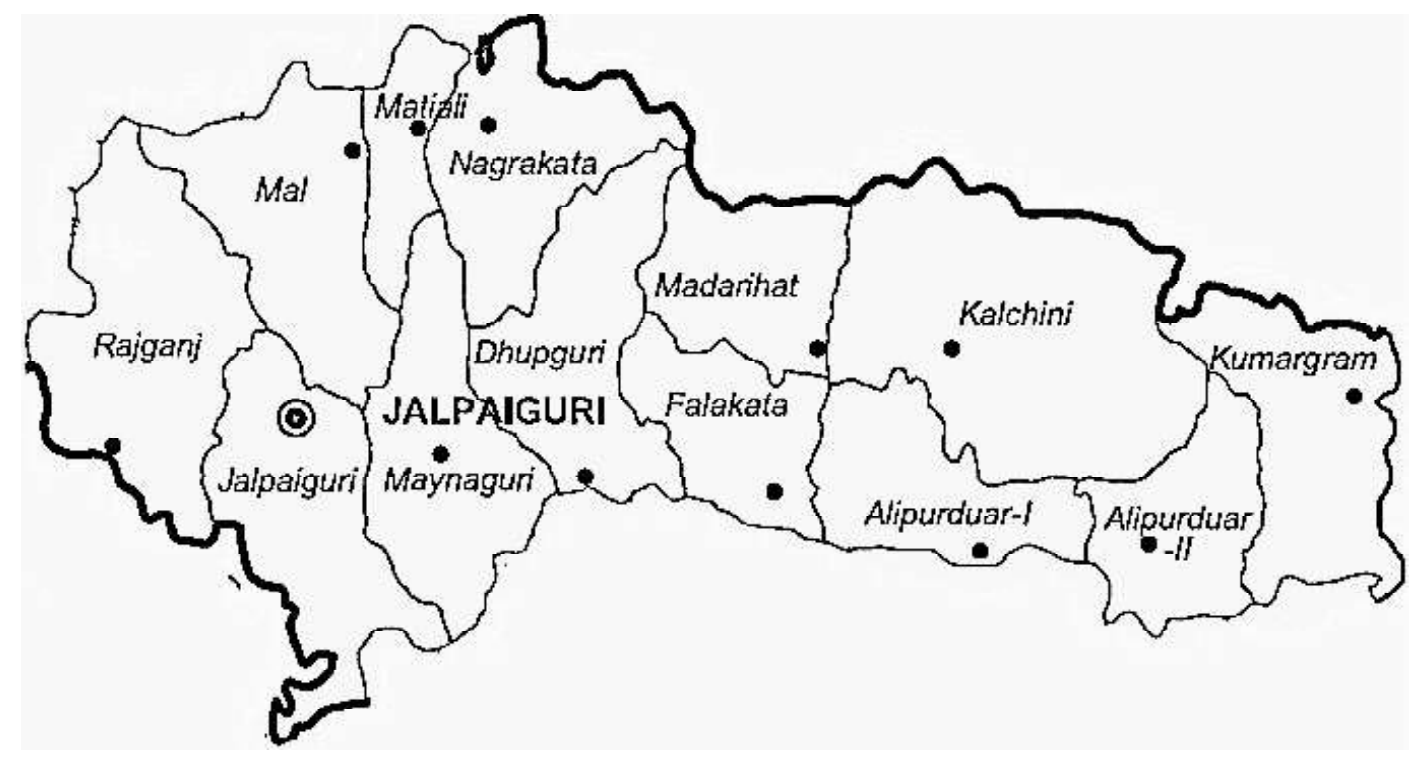

Figure 1. Location map of Jalpaiguri District [Source: https://images.app.goo.gl/ Mgf6YzP3NMadjGRz8]

\section{MATERIALS AND METHODS}

The traditional societies are, in general, very much conservative. Entire methodology was decided keeping that in mind and involving the people of the community in the entire exercise as it was followed by us in 2017 (Debnath et al. 2017). The survey was conducted during March 2017 - March 2019. 
Collected voucher specimens were processed into mounted Herbarium sheets and will be deposited to the NTFP Museum, Forest Department, Govt. of West Bengal, Sukna, Darjeeling, West Bengal. For the entire process Jain and Rao (1977) and Das (2018) has been followed. All the plant names and their family delimitations are checked in www.theplantlist.org.

\section{RESULT AND DISCUSSION}

Medicinal plants are used for cure of several diseases. Rajbangsi tribal people used many process of medicine preparation $\&$ used several plant parts for the preparation of medicine. In Table1, formulations of medicines, local and scientific name of plants, used plant parts, ratio of applied medicine and the names of diseases they treat are presented.

Table 1. Medicinal plants used by Rajbangsi people of Jalpaiguri District

\begin{tabular}{|c|c|c|c|c|c|c|}
\hline $\begin{array}{l}\text { Sl. } \\
\text { No. }\end{array}$ & $\begin{array}{l}\text { Formulation } \\
\text { in local name }\end{array}$ & Scientific name & $\begin{array}{l}\text { Parts } \\
\text { used }\end{array}$ & Preparation & Administration & $\begin{array}{l}\text { Disease } \\
\text { cures }\end{array}$ \\
\hline 1. & $\begin{array}{l}\text { Tulshi, Jol } \\
\text { (water) }\end{array}$ & $\begin{array}{l}\text { Ocimum tenuiflorum L. } \\
\text { [Labiatae ]; D.Deb- } 320\end{array}$ & Leaves & $\begin{array}{l}2 \text { leaves } \\
\text { squeezed in } \\
\text { c. } 8 \mathrm{ml} \text { of } \\
\text { water }\end{array}$ & Taken orally & Fever \\
\hline 2. & Aarahar & $\begin{array}{l}\text { Cajanus cajan (L.) } \\
\text { Millsp. [Leguminosae]; } \\
\text { D.Deb- } 421\end{array}$ & Leaves & $\begin{array}{l}\text { Extract in } \\
\text { water }\end{array}$ & $\begin{array}{l}1 \text { spoon in } \\
\text { empty stomach } \\
\text { in morning until } \\
\text { cures }\end{array}$ & Jaundice \\
\hline 3. & Ghritokumari & $\begin{array}{l}\text { Aloe vera (L.) Burm.f. } \\
\text { [Xanthorrhoeaceae]; } \\
\text { D.Deb- } 332\end{array}$ & Leaves & $\begin{array}{l}\text { Extract } \\
\text { from leaf- } \\
\text { jelly }\end{array}$ & $\begin{array}{l}10-15 \text { drops } \\
\text { on scalp }\end{array}$ & Hair fall \\
\hline 4. & Gulancha & $\begin{array}{l}\text { Tinospora sinensis } \\
\text { (Lour.) Merr. } \\
\text { [Menispermaceae]; } \\
\text { D.Deb- } 520\end{array}$ & Stem & $\begin{array}{l}\text { Extract } \\
\text { from } \\
\text { crushed } \\
\text { stem }\end{array}$ & $\begin{array}{l}1 / 2 \text { cup in empty } \\
\text { stomach }\end{array}$ & Diabetes \\
\hline 5. & $\begin{array}{l}\text { Basok, } \\
\text { Tulshi, Modhu } \\
\text { (honey) }\end{array}$ & $\begin{array}{l}\text { Justicia adhatoda L. } \\
\text { [Acanthaceae]; D.Deb- } \\
\text { 408.Ocimum tenuiflorum L } \\
\text { [Labiatae ]; D.Deb- } 320\end{array}$ & Leaves & $\begin{array}{l}\text { Extract in } \\
\text { little water } \\
+ \text { honey }\end{array}$ & $\begin{array}{l}2 \text { spoon, twice } \\
\text { or thrice daily }\end{array}$ & $\begin{array}{l}\text { Cold \& } \\
\text { cough }\end{array}$ \\
\hline 6. & Aam holud & $\begin{array}{l}\text { Curcuma longa L. } \\
\text { [Zingiberaceae]; D.Deb- } \\
233\end{array}$ & $\begin{array}{l}\text { Rhiz- } \\
\text { ome }\end{array}$ & $\begin{array}{l}\text { Paste in } \\
\text { water }\end{array}$ & $\begin{array}{l}1 / 3 \text { cup twice } \\
\text { or thrice daily }\end{array}$ & $\begin{array}{l}\text { Cold } \\
\text { cough }\end{array}$ \\
\hline 7. & Neem & $\begin{array}{l}\text { Azadirachta indica A. } \\
\text { Juss. [Meliaceae]; D.Deb- } \\
425\end{array}$ & Leaves & $\begin{array}{l}\text { Extract in } \\
\text { littlewater }\end{array}$ & $\begin{array}{l}2 \text { spoon in } \\
\text { empty stomach }\end{array}$ & $\begin{array}{l}\text { Skin } \\
\text { disease }\end{array}$ \\
\hline 8. & Dhulpi & $\begin{array}{l}\text { Leucas zeylanica }(\mathrm{L} .) \\
\text { W.T.Aiton [Labiatae]; } \\
\text { D.Deb- } 003\end{array}$ & Leaves & $\begin{array}{l}\text { Aquous } \\
\text { extract }\end{array}$ & $\begin{array}{l}1 \text { spoon twice/ } \\
\text { thrice daily till } \\
\text { cures }\end{array}$ & Pain \\
\hline 9. & Ganda & $\begin{array}{l}\text { Tagetes erecta } \mathrm{L} . \\
\text { [Compositae]; D.Deb- } 067\end{array}$ & Leaves & $\begin{array}{l}\text { Juice of } \\
\text { crushed leaf }\end{array}$ & $\begin{array}{l}\text { 8-9 drops } \\
\text { applied locally }\end{array}$ & Antiseptic \\
\hline 10 & Nishinda & $\begin{array}{l}\text { Vitex negundo L. } \\
\text { [Labiatae]; D.Deb- } 420\end{array}$ & Leaves & $\begin{array}{l}\text { Extract of } \\
\text { crushed leaf }\end{array}$ & $\begin{array}{l}2 \text { spoon daily; } \\
\text { applied locally } \\
\text { or consumed }\end{array}$ & $\begin{array}{l}\text { Wounds, } \\
\text { Jondis }\end{array}$ \\
\hline
\end{tabular}


58 Ethnomedicobotany of Rajbanshi community in Jalpaiguri District

\begin{tabular}{|c|c|c|c|c|c|c|}
\hline $\begin{array}{l}\text { Sl. } \\
\text { No. }\end{array}$ & $\begin{array}{l}\text { Formulation } \\
\text { in local name }\end{array}$ & Scientific name & $\begin{array}{l}\text { Parts } \\
\text { used }\end{array}$ & Preparation & Administration & $\begin{array}{l}\text { Disease } \\
\text { cures }\end{array}$ \\
\hline 11 & Vauti & $\begin{array}{l}\text { Clerodendrum } \\
\text { infortunatum L. } \\
\text { [Labiatae]; D.Deb- } 303\end{array}$ & Leaves & $\begin{array}{l}\text { Extract of } \\
\text { crushed leaf }\end{array}$ & Applied locally & $\begin{array}{l}\text { Wound } \\
\text { cure }\end{array}$ \\
\hline 12 & $\begin{array}{l}\text { Erenda, } \\
\text { Kola, Noon } \\
\text { (salt), Chini } \\
\text { (sugar) }\end{array}$ & $\begin{array}{l}\text { Jatropha curcas L. } \\
\text { [Euphorbiaceae]; D.Deb- } \\
\text { 032. } \\
\text { Musa x paradisiaca L. } \\
\text { [Musaceae]; D.Deb- } 202\end{array}$ & $\begin{array}{l}\text { Bulb, } \\
\text { fruits }\end{array}$ & $\begin{array}{l}\text { Crushed and } \\
\text { extracted } \\
\text { together }\end{array}$ & $\begin{array}{l}1 / 2 \text { cup taken } \\
\text { twice/ thrice } \\
\text { daily }\end{array}$ & Dysentery \\
\hline 13 & $\begin{array}{l}\text { Manimuni, } \\
\text { Golmorchi }\end{array}$ & $\begin{array}{l}\text { Centella asiatica }(\mathrm{L} .) \\
\text { Urb. [Umbelliferae]; } \\
\text { D.Deb- } 491 . \\
\text { Piper nigrum L. } \\
\text { [Piperaceae]; D.Deb- } 234\end{array}$ & $\begin{array}{l}\text { Leaves, } \\
\text { fruits }\end{array}$ & $\begin{array}{l}\text { Crushed and } \\
\text { extracted }\end{array}$ & $\begin{array}{l}1 \text { spoon } 2 \text { or } 3 \\
\text { times daily }\end{array}$ & Diarrhea \\
\hline 14 & $\begin{array}{l}\text { Athia kola, } \\
\text { Golmorchi }\end{array}$ & $\begin{array}{l}\text { Musa x paradisiaca } \mathrm{L} . \\
\text { [Musaceae]; D.Deb- } 202 . \\
\text { Piper nigrum L. } \\
\text { [Piperaceae]; D.Deb- } 234\end{array}$ & $\begin{array}{l}\text { Stem, } \\
\text { fruits }\end{array}$ & $\begin{array}{l}\text { Crushed and } \\
\text { extracted }\end{array}$ & $\begin{array}{l}1 \text { spoon } 2 \text { or } 3 \\
\text { times daily }\end{array}$ & Diarrhea \\
\hline 15 & $\begin{array}{l}\text { Peyara, } \\
\text { Golmorchi }\end{array}$ & $\begin{array}{l}\text { Psidium guajava L. } \\
\text { [Myrtaceae]; D.Deb- } 080 . \\
\text { Piper nigrum L. } \\
\text { [Piperaceae]; D.Deb- } 234\end{array}$ & $\begin{array}{l}\text { Imma- } \\
\text { ture } \\
\text { fruits }\end{array}$ & $\begin{array}{l}\text { Crushed and } \\
\text { extracted }\end{array}$ & $\begin{array}{l}1 \text { spoon } 2 \text { or } 3 \\
\text { times daily }\end{array}$ & Diarrhea \\
\hline 16 & $\begin{array}{l}\text { Tetul, } \\
\text { Golmorchi }\end{array}$ & $\begin{array}{l}\text { Tamarindus indica } \text { L. } \\
\text { [Leguminosae]; D.Deb- } \\
529 . \\
\text { Piper nigrum L. } \\
\text { [Piperaceae]; D.Deb- } 234\end{array}$ & Leaves & $\begin{array}{l}\text { Crushed and } \\
\text { extracted }\end{array}$ & $\begin{array}{l}1 \text { spoon } 2 \text { or } 3 \\
\text { times daily }\end{array}$ & Diarrhea \\
\hline 17 & $\begin{array}{l}\text { Kathol, } \\
\text { Golmorchi }\end{array}$ & $\begin{array}{l}\text { Artocarpus heterophyllus } \\
\text { Lam. [Moraceae]; } \\
\text { D.Deb- } 517 \text {. } \\
\text { Piper nigrum L. } \\
\text { [Piperaceae]; D.Deb- } 234\end{array}$ & Fruits & $\begin{array}{l}\text { Crushed and } \\
\text { extracted }\end{array}$ & $\begin{array}{l}1 \text { spoon } 2 \text { or } 3 \\
\text { times daily }\end{array}$ & Diarrhea \\
\hline 18 & $\begin{array}{l}\text { Paan, } \\
\text { Aada }\end{array}$ & $\begin{array}{l}\text { Piper betle L. } \\
\text { [Piperaceae]; D.Deb- } 305 . \\
\text { Zingiber officinale } \\
\text { Roscoe [Zingiberaceae]; } \\
\text { D.Deb- } 223\end{array}$ & $\begin{array}{l}\text { Leaves, } \\
\text { stem }\end{array}$ & $\begin{array}{l}\text { Crushed and } \\
\text { extracted }\end{array}$ & $\begin{array}{l}1 \text { spoon in the } \\
\text { morning or } \\
\text { SOS }\end{array}$ & Gastric \\
\hline 19 & Anarosh & $\begin{array}{l}\text { Ananas comosus (L.) } \\
\text { Merr. [Bromeliaceae]; } \\
\text { D.Deb- } 381\end{array}$ & Leaves & $\begin{array}{l}\text { Extract of } \\
\text { basal soft } \\
\text { portion }\end{array}$ & $\begin{array}{l}1 / 2 \text { cup in the } \\
\text { morning }\end{array}$ & $\begin{array}{l}\text { Wound } \\
\text { cure }\end{array}$ \\
\hline 20 & Kola & $\begin{array}{l}\text { Musa } \times \text { paradisiaca L. } \\
\text { [Musaceae]; D.Deb- } 202\end{array}$ & Leaves & $\begin{array}{l}\text { Squeezed } \\
\text { jice }\end{array}$ & $\begin{array}{l}2 \text { spoon } \\
\text { applied locally }\end{array}$ & $\begin{array}{l}\text { Dental } \\
\text { wound }\end{array}$ \\
\hline 21 & $\begin{array}{l}\text { Nebu, Narkel } \\
\text { tel [coconut } \\
\text { oil], Jol }\end{array}$ & $\begin{array}{l}\text { Citrus aurantiifolia } \\
\text { (Christm.) Swingle } \\
\text { [Rutaceae]; D.Deb- } 309 . \\
\text { Cocos nucifera L. } \\
\text { [Arecaceae]; D.Deb- } 350\end{array}$ & Leaves & $\begin{array}{l}\text { Leaf-extract } \\
\text { with oil \& } \\
\text { water }\end{array}$ & $1 / 2$ cup in SOS & Headache \\
\hline
\end{tabular}



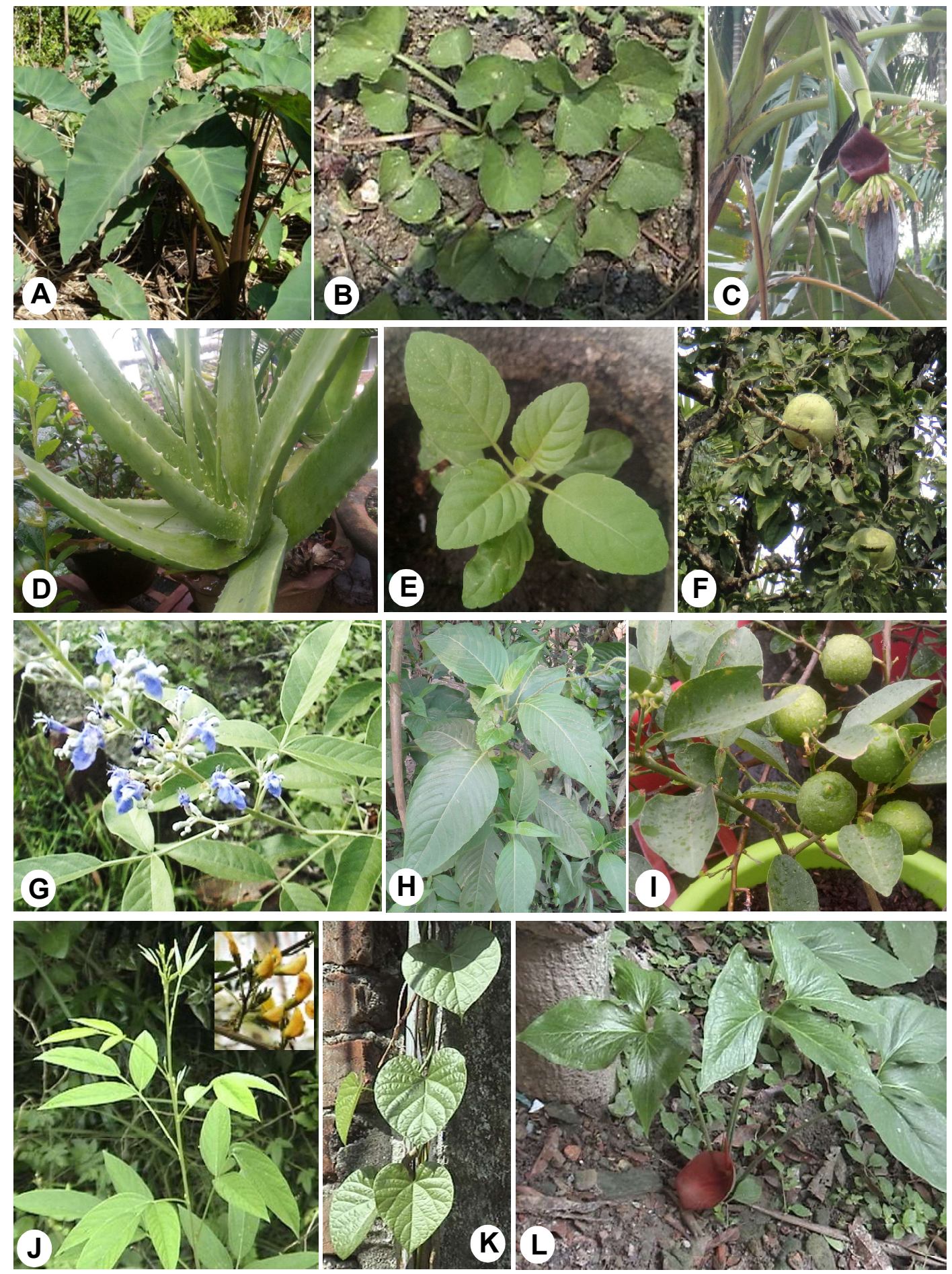

PLATE - I. A. Colocasia esculenta; B. Centella asiatica; C. Musa x paradisiaca; D. Aloe vera; E. Ocimum tenuiflorum; F. Aegle marmelos: G. Vitex negundo; H. Justicia adhatoda; I. Citrus aurantiifolia; J. Cajanus cajan; K. Tinospora sinensis; L. Typhonium trilobatum 
60 Ethnomedicobotany of Rajbanshi community in Jalpaiguri District

\begin{tabular}{|c|c|c|c|c|c|c|}
\hline $\begin{array}{l}\text { Sl. } \\
\text { No. }\end{array}$ & $\begin{array}{l}\text { Formulation } \\
\text { in local name }\end{array}$ & Scientific name & $\begin{array}{l}\text { Parts } \\
\text { used }\end{array}$ & Preparation & Administration & $\begin{array}{l}\text { Disease } \\
\text { cures }\end{array}$ \\
\hline 22 & Holud & $\begin{array}{l}\text { Curcuma longa } \mathrm{L} \text {. } \\
\text { [Zingiberaceae]; D.Deb- } \\
233\end{array}$ & $\begin{array}{l}\text { Pseudos } \\
\text { tem }\end{array}$ & $\begin{array}{l}\text { Extracted } \\
\text { juice }\end{array}$ & $\begin{array}{l}2-3 \text { drops in } \\
\text { eye }\end{array}$ & $\begin{array}{l}\text { Opthalmia, } \\
\text { Conjunctiv- } \\
\text { itis }\end{array}$ \\
\hline 23 & $\begin{array}{l}\text { Nishinda, } \\
\text { Nim, } \\
\text { Malvog kola }\end{array}$ & $\begin{array}{l}\text { Vitex negundo L. } \\
\text { [Labiatae]; D.Deb- } 420 \text {. } \\
\text { Azadirachta indica A. } \\
\text { Juss. [Meliaceae]; } \\
\text { D.Deb- } 518 . \text { Musa x } \\
\text { paradisiaca L. } \\
\text { [Musaceae]; D.Deb- } 202\end{array}$ & $\begin{array}{l}\text { Leaves, } \\
\text { stem }\end{array}$ & Extract & $\begin{array}{l}\text { Applied on the } \\
\text { body }\end{array}$ & $\begin{array}{l}\text { Chicken } \\
\text { Pox }\end{array}$ \\
\hline 24 & $\begin{array}{l}\text { Gom (flour } \\
\text { scraps), } \\
\text { Khorimati \& } \\
\text { Hukkkah } \\
\text { (waterpipe) Jol }\end{array}$ & $\begin{array}{l}\text { Triticum aestivum L. } \\
\text { [Gramineae]; D.Deb- } 441\end{array}$ & $\begin{array}{l}\text { Seed } \\
\text { coat }\end{array}$ & Powdered & Appled locally & Scabies \\
\hline 25 & $\begin{array}{l}\text { Tulshi, Joba, } \\
\text { Aam Holud, } \\
\text { Noon }\end{array}$ & $\begin{array}{l}\text { Ocimum tenuiflorum L. } \\
\text { [Labiatae]; D.Deb- } 320 . \\
\text { Hibiscus rosa-sinensis L. } \\
\text { [Malvaceae]; D.Deb- } 007 \text {. } \\
\text { Curcuma longa L. } \\
\text { [Zingiberaceae]; D.Deb- } \\
233\end{array}$ & $\begin{array}{l}\text { Leaves, } \\
\text { flowers }\end{array}$ & $\begin{array}{l}\text { Concentrate } \\
\text { d extract of } \\
\text { all }\end{array}$ & $\begin{array}{l}3 \text { spoon } \\
\text { consumed and } \\
\text { applied locally }\end{array}$ & $\begin{array}{l}\text { Ring } \\
\text { worm }\end{array}$ \\
\hline 26 & Nebu & $\begin{array}{l}\text { Citrus aurantiifolia } \\
\text { (Christm.) Swingle } \\
\text { [Rutaceae]; D.Deb- } 309\end{array}$ & Leaves & Dry crush & $\begin{array}{l}\text { Inhale aroma } \\
\text { on SOS }\end{array}$ & Vomiting \\
\hline 27 & Alok lota & $\begin{array}{l}\text { Cuscuta reflexa Roxb. } \\
\text { [Convolvulaceae]; } \\
\text { D.Deb- } 506\end{array}$ & $\begin{array}{l}\text { Yellow } \\
\text { stem }\end{array}$ & $\begin{array}{l}\text { Extract with } \\
\text { little water }\end{array}$ & $\begin{array}{l}1 / 2 \text { cup taken } \\
\text { orally }\end{array}$ & $\begin{array}{l}\text { Profuse } \\
\text { bleeding } \\
\text { during } \\
\text { delivary }\end{array}$ \\
\hline 28 & $\begin{array}{l}\text { Makla Bansh } \\
\text { \& Chun [lime] }\end{array}$ & $\begin{array}{l}\text { Bambusa tulda Roxb. } \\
\text { [Gramineae]; D.Deb- } 401\end{array}$ & $\begin{array}{l}\text { Outer } \\
\text { coat of } \\
\text { stem }\end{array}$ & $\begin{array}{l}\text { Scrapped to } \\
\text { make a } \\
\text { powder }\end{array}$ & Applied locally & $\begin{array}{l}\text { Deep } \\
\text { wound }\end{array}$ \\
\hline 29 & Kalo kechrai & $\begin{array}{l}\text { Andrographis paniculata } \\
\text { (Burm.f.) Nees } \\
\text { [Acanthaceae]; D.Deb- } 571\end{array}$ & Leaves & $\begin{array}{l}\text { Made into } \\
\text { paste }\end{array}$ & Applied locally & Burn spot \\
\hline 30 & $\begin{array}{l}\text { Gha chatar } \\
\text { pata }\end{array}$ & $\begin{array}{l}\text { Typhonium trilobatum (L.) } \\
\text { Schott [Araceae]; D.Deb- } \\
439\end{array}$ & Leaves & $\begin{array}{l}\text { Made into } \\
\text { paste }\end{array}$ & Applied locally & Boils \\
\hline 31 & Kochu & $\begin{array}{l}\text { Arum maculatum L. } \\
\text { [Araceae]; D.Deb- } 329\end{array}$ & Leaves & $\begin{array}{l}\text { Made into } \\
\text { paste }\end{array}$ & Applied locally & Infection \\
\hline 32 & $\begin{array}{l}\text { Sajina, Ashuni } \\
\text { saakh, } \\
\text { Oshun, } \\
\text { Golmorchi }\end{array}$ & \begin{tabular}{|l} 
Moringa oleifera Lam. \\
{$[$ Moringaceae]; D.Deb- 200.} \\
Marsilea minuta L. \\
[Marsileaceae]; D.Deb- 311. \\
Piper nigrum L. Piperaceae] \\
D.Deb- 234
\end{tabular} & $\begin{array}{l}\text { Stem, } \\
\text { fruits }\end{array}$ & $\begin{array}{l}\text { Crushed and } \\
\text { extracted }\end{array}$ & $\begin{array}{l}1 \text { Cup in the } \\
\text { morning till } \\
\text { cures }\end{array}$ & Anaemia \\
\hline
\end{tabular}




\begin{tabular}{|c|c|c|c|c|c|c|}
\hline $\begin{array}{l}\text { SI. } \\
\text { No. }\end{array}$ & $\begin{array}{l}\text { Formulation } \\
\text { in local name }\end{array}$ & Scientific name & $\begin{array}{l}\text { Parts } \\
\text { used }\end{array}$ & Preparation & Administration & $\begin{array}{l}\text { Disease } \\
\text { cures }\end{array}$ \\
\hline 33 & Bansh & $\begin{array}{l}\text { Bambusa tulda Roxb. } \\
\text { [Gramineae]; D.Deb- } 401\end{array}$ & Leaves & $\begin{array}{l}\text { Made into } \\
\text { paste }\end{array}$ & Applied locally & Snake bite \\
\hline 34 & Shukati & $\begin{array}{l}\text { Corchorus capsularis L. } \\
\text { [Malvaceae]; D.Deb- } 301\end{array}$ & Dry leaf & $\begin{array}{l}\text { Made into } \\
\text { paste }\end{array}$ & Applied locally & $\begin{array}{l}\text { Wound } \\
\text { with Singi } \\
\text { fish }\end{array}$ \\
\hline 35 & Nishinda & $\begin{array}{l}\text { Vitex negundo L. } \\
\text { [Labiatae]; D.Deb- } 420\end{array}$ & Leaves & $\begin{array}{l}\text { Crushed and } \\
\text { extracted }\end{array}$ & $\begin{array}{l}2 \text { spoonful in } \\
\text { the morning }\end{array}$ & $\begin{array}{l}\text { Prevent } \\
\text { whitening } \\
\text { of hair, } \\
\text { improves } \\
\text { memory }\end{array}$ \\
\hline 36 & Aam & $\begin{array}{l}\text { Mangifera indica } \text { L. } \\
\text { [Anacardiaceae]; D.Deb- } \\
198\end{array}$ & $\begin{array}{l}\text { Gum } \\
\text { from } \\
\text { bark }\end{array}$ & & Applied locally & $\begin{array}{l}\text { Cracked } \\
\text { foot }\end{array}$ \\
\hline 37 & Kalo kochu & $\begin{array}{l}\text { Colocasia esculenta }(\mathrm{L} .) \\
\text { Schott [Araceae]; D.Deb- } \\
512\end{array}$ & Stem & $\begin{array}{l}\text { Made into } \\
\text { paste }\end{array}$ & Applied locally & Gurguchia \\
\hline 38 & $\begin{array}{l}\text { Holud, Vui } \\
\text { kumra, } \\
\text { chhyaka }\end{array}$ & $\begin{array}{l}\text { Curcuma longa L. } \\
\text { [Zingiberaceae]; D.Deb- } \\
\text { 233. Cucurbita maxima } \\
\text { Duchesne [Cucurbitaceae]; } \\
\text { D.Deb- } 312\end{array}$ & $\begin{array}{l}\text { Rhizom } \\
\text { e, stem }\end{array}$ & Extract & 1 cup SOS & $\begin{array}{l}\text { Pet kamri } \\
\text { [stomach- } \\
\text { ache] }\end{array}$ \\
\hline 39 & Paan & $\begin{array}{l}\text { Piper betle L. } \\
\text { [Piperaceae]; D.Deb- } 305\end{array}$ & Leaves & Extract & SOS & Flatulence \\
\hline 40 & $\begin{array}{l}\text { Bara } \\
\text { Manimuni }\end{array}$ & $\begin{array}{l}\text { Centella asiatica }(\mathrm{L} .) \\
\text { Urb. [Umbelliferae]; } \\
\text { D.Deb- } 491\end{array}$ & Leaves & Extract & $\begin{array}{l}\text { Taken in empty } \\
\text { stomach in } \\
\text { morning }\end{array}$ & Dysentery \\
\hline 41 & $\begin{array}{l}\text { Kaach kola, } \\
\text { Chun, Modhu, } \\
\text { Noon }\end{array}$ & $\begin{array}{l}\text { Musa } \times \text { paradisiaca L. } \\
\text { [Musaceae]; D.Deb- } 202\end{array}$ & Fruits & Paste & $\begin{array}{l}\text { Applied on } \\
\text { forehead }\end{array}$ & Headache \\
\hline 42 & $\begin{array}{l}\text { Kena ghaas, } \\
\text { Aada, salt }\end{array}$ & $\begin{array}{l}\text { Actinoscirpus grossus } \\
\text { var. kysoor (Roxb.) Noltie } \\
\text { [Cyperaceae]; D.Deb- } \\
\text { 399. Zingiber officinale } \\
\text { Roscoe [Zingiberaceae]; } \\
\text { D.Deb- } 223\end{array}$ & $\begin{array}{l}\text { Root- } \\
\text { stock, } \\
\text { rhizome }\end{array}$ & Extract & $\begin{array}{l}1 \text { spoon on } \\
\text { SOS }\end{array}$ & $\begin{array}{l}\text { Pain in the } \\
\text { abdomen }\end{array}$ \\
\hline 43 & Kesari ghaas & $\begin{array}{l}\text { Lathyrus sativus L. } \\
\text { [Leguminosae]; D.Deb- } \\
386\end{array}$ & Roots & Paste & Applied locally & Wounds \\
\hline 44 & $\begin{array}{l}\text { Aalu [raw, } \\
\text { crushed] }\end{array}$ & $\begin{array}{l}\text { Solanum tuberosum L. } \\
\text { [Solanaceae]; D.Deb- } 377\end{array}$ & Tuber & $\begin{array}{l}\text { Crushed } \\
\text { raw }\end{array}$ & $\begin{array}{l}\text { Applied } \\
\text { locally }\end{array}$ & Burns \\
\hline 45 & Hemkatsa & $\begin{array}{l}\text { Bryophyllum pinnatum } \\
\text { (Lam.) Oken } \\
\text { [Crassulaceae]; D.Deb- } 363\end{array}$ & Leaves & $\begin{array}{l}\text { Made into } \\
\text { paste }\end{array}$ & Applied locally & Burns \\
\hline
\end{tabular}


Ethnomedicobotany of Rajbanshi community in Jalpaiguri District

\begin{tabular}{|c|c|c|c|c|c|c|}
\hline $\begin{array}{l}\text { SI. } \\
\text { No. }\end{array}$ & $\begin{array}{l}\text { Formulation } \\
\text { in local name }\end{array}$ & Scientific name & $\begin{array}{l}\text { Parts } \\
\text { used }\end{array}$ & Preparation & Administration & $\begin{array}{l}\text { Disease } \\
\text { cures }\end{array}$ \\
\hline 46 & $\begin{array}{l}\text { Muri [parched } \\
\text { rice], Dhup } \\
\text { [incense stick, } \\
\text { white], Noon }\end{array}$ & $\begin{array}{l}\text { Oryza sativa L. } \\
\text { [Gramineae]; D.Deb- } 209\end{array}$ & Fruits & $\begin{array}{l}\text { Mixed and } \\
\text { powdered }\end{array}$ & $\begin{array}{l}2 \text { Cups } 2 \text { or } 3 \\
\text { times daily }\end{array}$ & Diarrhoea \\
\hline 47 & Dhonia & $\begin{array}{l}\text { Coriandrum sativum L. } \\
\text { [Umbelliferae]; D.Deb- } \\
199\end{array}$ & Fruits & Powdered & $\begin{array}{l}1 / 2 \text { spoon after } \\
\text { meals }\end{array}$ & $\begin{array}{l}\text { Indigest- } \\
\text { ion, } \\
\text { vomiting }\end{array}$ \\
\hline 48 & Bel & $\begin{array}{l}\text { Aegle marmelos (L.) } \\
\text { Correa [Rutaceae]; } \\
\text { D.Deb- } 319\end{array}$ & $\begin{array}{l}\text { Fruits, } \\
\text { seeds }\end{array}$ & $\begin{array}{l}\text { Made into } \\
\text { extract }\end{array}$ & $\begin{array}{l}\text { Need to take } \\
\text { regularly }\end{array}$ & $\begin{array}{l}\text { Stomach } \\
\text { problem }\end{array}$ \\
\hline 49 & Dhutra & $\begin{array}{l}\text { Datura stramonium L. } \\
\text { [Solanaceae]; D.Deb- } 314\end{array}$ & Seeds & Powdered & $\begin{array}{l}\text { Applied } \\
\text { locally }\end{array}$ & Dog bite \\
\hline 50 & Pui shaak & $\begin{array}{l}\text { Basella alba L. } \\
\text { [Basellaceae]; D.Deb- } 313\end{array}$ & Roots & Extract & $\begin{array}{l}2 \text { or } 3 \text { times } \\
\text { daily }\end{array}$ & Tuberculosis \\
\hline
\end{tabular}

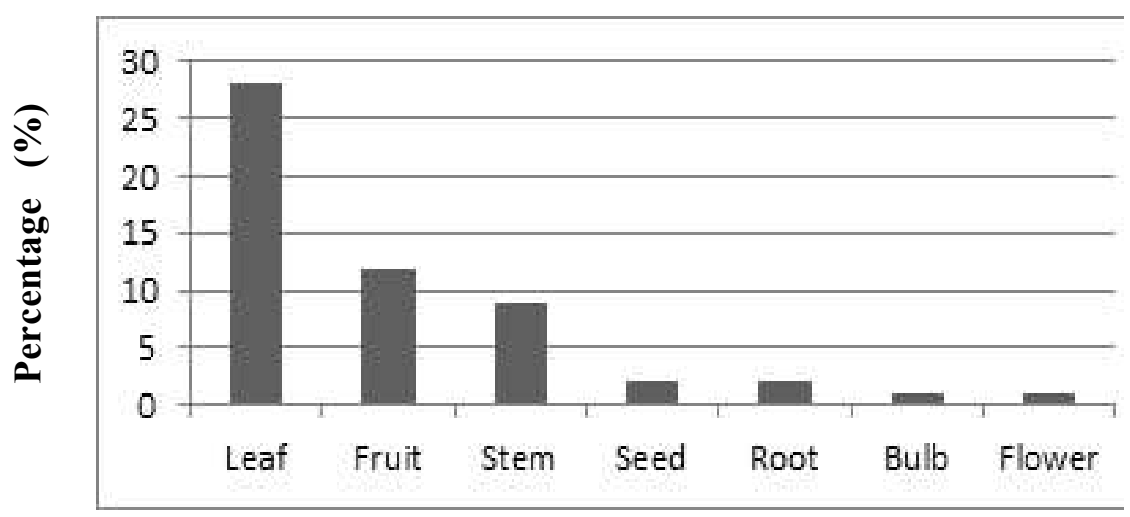

Plant parts used

Figure 2. Percentage of plant parts used as medicine by the Rajbangsi community in Jalpaiguri district of West Bengal

From this above table it is observed that majority of their traditional medicines are prepared from leaves and the least number of medicines prepared from flowers. Percentage of plant parts used as medicine by Rajbangsi community in Jalpaiguri district of West Bengal is shown graphically in Figure 2.

Out of the 50 formulations presented in Table 1, as much as 36 are with single species of plants. For the remaining 14 formulations they are using $2-4$ plant species. Interestingly, except Marsilea minuta all other medicinal plants recorded during the present survey are angiospermic. And, only 13 of those are monocotyledonous. Apart from plant parts, the 
record of the uses of salt, sugar and lime was used. In majority of the formulations water has been used to make extract, decoction, etc. Another tribal community, Mech, living in the same area, has developed better practice in formulation and preparation of medicine. Even, they prepare tablets sometimes with more than 10 species (Sarkar 2011; Sarkar \& Das 2010, 2012). However, there is another probability as educationally Rajbanshis are more advanced and are also enjoing the facilities of modern civilization for a longer period. In such case may be their traditional medican knowledge is being affected by the alopathic systems.

On search, no written traditional document was available with them for medical treaments among the Rajbanshi people. So, their traditional knowledge of medicine in the study area was transmitted orally for generations. But, the extension of modern facilities through tea-planters' and through the activities of Government Forest Department people in the Terai - Duars region brought them closure to such facilities. It is also noticed that younger generation of Rajbangsi people have less amount of knowledge about their tradition. So, it is now extremely important to documentation of their all types of traditional knowledge, including medicinal, otherwise their traditional knowledge will lost completely within next few years.

\section{CONCLUSION}

Not only modern medical facilities, almost all other facilities of modern civilisation are available in most part of the northern districts of West Bengal. Regular medical camps by government and by NGO's also helping them to avail such facilities. Facilities of modern education through the establishment of numerous schools, colleges and universities are enlighting these people very fast. So, not only for Rajbanshi's or Mech's, but for all other traditional communities living in this area need to be surveyed immediately to record their all traditional knowledge for future scientific evaluation considering the benefits of the humanity.

\section{Acknowlwdgements}

The authors are really thankful to the local people of different areas of Jalpaiguri district for sharing their traditional knowledge as they realised it through logical interactions.

\section{LITERATURE CITED}

Das, A.P. 2018. Herbarium Techniques. [In: J.B. Bhandari \& Cyria Gurung, Instrumentation Mannual. Bishen Singh Mahendra Pal Singh, Dehradun. in press]

Debnath, D; Choudhury,D; Chowdhury,M \& Das, A.P. 2017. Ethnobotany of Rajbanshi Community in Duars of Jalpaiguri District, West Bengal, India: 1. Traditional food. Pleione 11(1): 137-145.

Jain, S.K. \& Rao, R.R.1977. A Handbook of Field and Herbarium Methods. Today \& Tomorrow Printers and Publishers, New Delhi.

Mukherjee, S.K.; Das, A.P. \& Bera, Subir 2007. Ethnic uses of honey in Sikkim and subHimalayan West Bengal, India. In: Das, A.P. \& Pandey, A.K. (eds.), Advances in Ethnobotany. Bishen Singh Mahindra Pal Singh, Dehradun. Pp. 189 - 197.

Roy, S. \& Das, A.P. 2015. Some favourite Rajbanshi cuisine from the northern part of West Bengal, India. Pleione 9(2): $471-480$.

Sanyal, C.C. 1965. The Rajbangsi's of North Bengal. The Asiatic Society, Kolkata.

Sarkar, A. 2011. Ethnobotany of Mech Tribe in North Bengal and Assam. Ph.D. thesis, University of North Bengal, Siliguri. 
64 Ethnomedicobotany of Rajbanshi community in Jalpaiguri District

Sarkar, Ajita \& Das, A.P. 2012. Mech's way of treatment of some respiratory diseases in the northern districts of West Bengal, India. In: G.G. Maiti \& S.K. Mukherjee (eds.), International Seminar on "Multidisciplinary Approaches in Angiospenn Systematics". University of Kalyani, Kalyani. Pp. 625-632.

Sarkar, Ajita \& Das, A.P. 2010. Ethnobotanical formulations for the treatment ofJaundice by the Mech tribe in Duars of West Bengal. Indian J. Trad. Knowl. 9 (1) : $134-136$.

www.rajbanshipride.blogspot.in/2016/02/social-issiues-scst-status-of-rajbanshi

www.theplantlist.org 